\title{
Effect of Extrinsic and Intrinsic Stressors on Clinical Skills Performance in Third-Year Medical Students
}

\author{
Pierre Pottier, MD PhD ${ }^{1,2}$, Jean-Benoit Hardouin, Ph.D. ${ }^{2}$, Thomas Dejoie, M.D. ${ }^{3}$, \\ Jean-Marie Castillo, M.D. ${ }^{4}$, Anne-Gaelle Le Loupp, M.D. ${ }^{3}$, Bernard Planchon, M.D. Ph.D. ${ }^{7}$, \\ Angélique Bonnaud, Ph.D. ${ }^{2,5}$, and Vicki LeBlanc, Ph.D.6
}

\begin{abstract}
'Department of Internal Medicine, University Hospital Center, Faculty of Medicine, University of Nantes, Nantes, France; ${ }^{2}$ EA4275, Biostatistics, Pharmacoepidemiology and Human Sciences Research, University of Nantes, Nantes, France; ${ }^{3}$ Laboratory of Biochemistry, University Hospital Center, Faculty of Medicine, University of Nantes, Nantes, France; ${ }^{4}$ Department of Family Medicine, Faculty of Medicine, University of Nantes, Nantes, France; ${ }^{5}$ Department of Social Sciences, Faculty of Medicine, University of Nantes, Nantes, France; ${ }^{6}$ Wilson Center for Research in Medical Education, University of Toronto, Toronto, Canada.
\end{abstract}

BACKGROUND: Both enhancements and impairments of clinical performance due to acute stress have been reported, often as a function of the intensity of an individual's response. According to the broader stress literature, peripheral or extrinsic stressors (ES) and task-contingent or intrinsic stressors (IS) can be distinguished within a stressful situation. The objective of this study was to assess the impact of IS and ES on clinical performance.

METHOD: A prospective randomized crossover study was undertaken with third-year medical students conducting two medical experiences with simulated patients. The effects of severity of the disease (IS) and the patient's aggressiveness (ES) were studied. A total of 109 students were assigned to four groups according to the presence of ES and IS. Subjective stress and anxiety responses were assessed before and after each experience. The students' clinical skills, diagnostic accuracy and argumentation were assessed as clinical performance measures. Sex and student-perceived cognitive difficulty of the task were considered as adjustment variables.

RESULTS: Both types of stressors improved clinical performance. IS improved diagnostic accuracy (regression parameter $\beta=9.7, p=0.004$ ) and differential argumentation ( $\beta=5.9, p=0.02)$, whereas ES improved clinical examination $(\beta=12.3, p<0.001)$ and communication skills $(\beta=15.4, p<0.001)$. The student-perceived cognitive difficulty of the task was a strong deleterious factor on both stress and performance.

CONCLUSION: In simulated consultation, extrinsic and intrinsic stressors both have a positive but different effect on clinical performance.

KEY WORDS: Stress; Clinical reasoning assessment; Medical students; Performance; Medical education research.

J Gen Intern Med 30(9): 1259-69

DOI: $10.1007 / \mathrm{s} 11606-015-3314-6$

(c) Society of General Internal Medicine 2015

\section{INTRODUCTION}

Medical students are exposed to many situations during which they need to make important clinical decisions while feeling

Published online July 15, 2015 stressed or anxious. ${ }^{1}$ In such situations, their ability to take a medical history, perform a physical examination, and engage in clinical reasoning could be influenced by their emotional state.

According to Lazarus, ${ }^{2}$ stress (or distress) is experienced by an individual when the perceived demands of a situation exceed the perceived available resources to meet these demands. Conversely, a challenge feeling (eustress) appears when the perceived resources meet or exceed the perceived demands of the situation.

The effect of acute stress on clinical reasoning remains unclear. Most of the studies looking at the effects of stress have focused on the cognitive functions of attention, memory or decision-making in laboratory settings. ${ }^{3-5}$ The broader stress literature suggests an impact on memory retrieval and working memory according to the intensity of stress, with eustress being beneficial to performance compared to no stress or distress. ${ }^{6,7}$ In the relatively few studies conducted with health professionals, both enhancements and impairments have been reported, generally also as a function of the subjective and physiological responses to acute situations. ${ }^{3,8}$ Some data suggest that stress responses could induce premature closure in one's initial hypothesis, ${ }^{9,10}$ which could lead to diagnostic errors. ${ }^{11}$

There is limited research suggesting that intrinsic stressors (integral —or tied — to the task being performed) could affect performance differently from extrinsic stressors (peripheral to the task being performed). When feeling stressed or anxious, a person's cognitive resources will be biased towards the perceived source of threat. ${ }^{12}$ If the stressors are intrinsic to the task being performed, this results in the person's attention and other cognitive resources being focused on the task. If, however, the stressors are extrinsic to the task, cognitive resources will be oriented towards those stressors at the expense of the task being performed. As such, integral stressors may facilitate performance by focusing attentional and other cognitive resources towards the task being performed. ${ }^{3}$ In the healthcare setting, extrinsic stressors (ES) can include noise and alarms, ${ }^{13}$ pain, ${ }^{14}$ disruptive participants (family member, colleague, team member), ${ }^{15}$ discord between participants, ${ }^{13,15}$ sleep 
deprivation, ${ }^{16}$ and social evaluation. ${ }^{17}$ In contrast, intrinsic stressors (IS) can include having to break bad news, ${ }^{18,19}$ high-stakes and urgent tasks, ${ }^{13,20-22}$ dealing with uncertainty, ${ }^{23}$ and novel tasks. ${ }^{10}$ To date, there has been no research in health professional education comparing the effects of extrinsic and intrinsic stressors within the same cohort of learners.

The goal of this study was to compare the effects of extrinsic and intrinsic stressors on medical students' clinical abilities (taking a history and performing a physical examination) and clinical reasoning. A secondary goal of this study was to examine the effects of perceived task difficulty independent of the effects of extrinsic and intrinsic stressors. We hypothesized that intrinsic stressors would enhance performance.

\section{METHODS}

\section{Design of the Study (see Fig. 1)}

This was a prospective crossover study, with medical students conducting two medical ambulatory experiences with standardized patients (SPs). Volunteer third-year medical students were recruited through oral presentations made at the end of several large group lectures at the beginning of the academic year (September 2011) so that all the third-year students were aware of the study protocol. Written consent was provided and was collected from September to December 2011. The SP sessions were scheduled from February 20 to March 2, 2012. All students, regardless of their participation in the study, attended one SP session, which included four simulated experiences.

Frequent and previously taught medical problems were chosen (scenario A, dyspnea due to pulmonary embolism; scenario B, abdominal pain due to pancreatitis). Students had to conduct a clinical exam and generate diagnostic hypotheses based on the clinical data obtained during the clinical evaluation.

Students were not aware of the stress conditions they would encounter in their experiences. Before they were exposed to the two study experiences, the students performed two training consultations in order to become habituated to the task and to minimize the novelty effects of the situation (this was their first-ever SP session). Each experience lasted $15 \mathrm{~min}$, with 5 min between each scenario.

The medical students' subjective stress responses were assessed three times: $5 \mathrm{~min}$ before the first study experience (baseline), immediately after the first study experience (Time 2 , or T2), and after the second study experience (Time 3, or T3).

\section{Ethical Considerations}

In the absence of ethics committees, for medical education research in France, the study protocol was read and approved by the dean of our faculty. The study protocol was also presented to a team of researchers in medical education at the Wilson Center in Toronto, Canada, for ethical review before the study was implemented. After receiving information regarding the details of the study protocol, students signed written consent forms. Data were analyzed anonymously. There was no incentive for participation. All students participated in the scenarios for the purpose of their education, but we collected data only from students who had consented to participate in the study.

\section{Stressors}

Extrinsic stressors were defined as stressful components peripheral to the task. Intrinsic stressors were defined as stressful components integral to the task itself. The task consisted of solving a clinical problem in a simulated ambulatory context.

Intrinsic Stressors (IS). As previously demonstrated, ${ }^{13,20}$ "clinical severity" was chosen as an intrinsic stressor and included in scenario A (pulmonary embolism). Students were tasked with determining the correct diagnosis when faced with a patient presenting with acute dyspnea in the context of disseminated malignancy and palliative treatment.

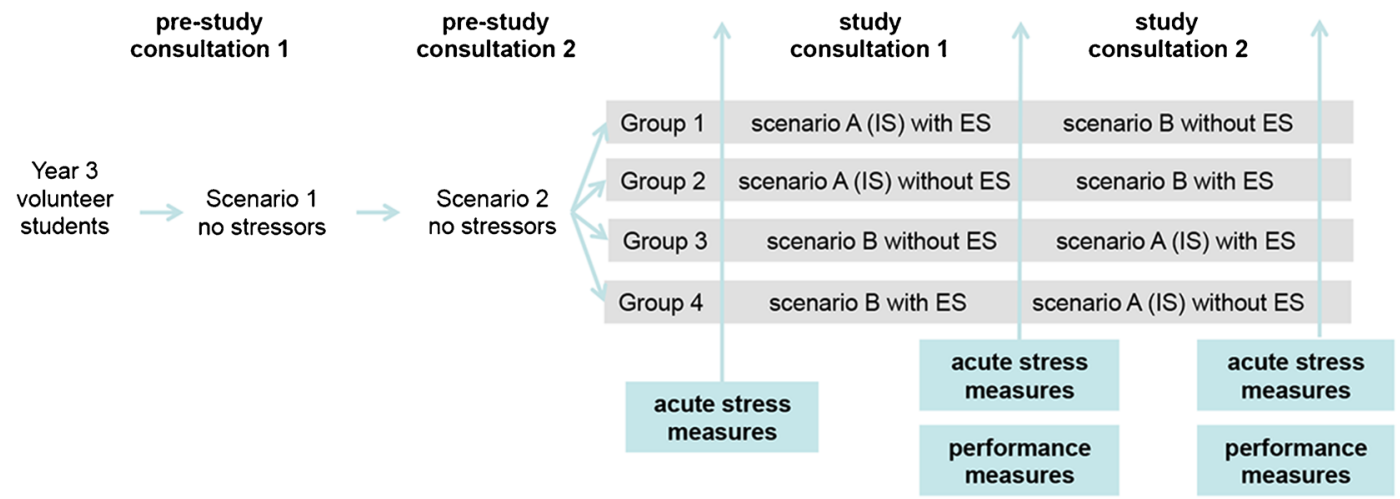

Fig. 1 Design of the study. Acute stress measures: cognitive appraisal, visual analogical scale, State-Trait Anxiety Inventory. Performance measures: ability to perform a clinical examination, communication skills, diagnostic accuracy, diagnostic argumentation. ES extrinsic stressor, $I S$ intrinsic stressor 
Clinical severity was manifested such that it was immediately recognized by the student (high respiratory rate $>$ 30 cycles/min and extreme difficulty talking). In no case was scenario A presented without signs of increased clinical severity. Moreover, the patient was aware of the diagnosis of cancer with metastatic dissemination and consequently expressed knowledge of the imminence of death. In addition to dyspnea, the patient was complaining of leg pain that he was certain was a new metastatic localization. He was also experiencing extreme fatigue. This particular condition was called "high clinical severity". Conversely, scenario B had no such intrinsic stressor and was called "low clinical severity". Scenario B consisted of a complaint of acute abdominal pain in a relatively healthy patient without any serious underlying pathology.

Extrinsic Stressors (ES). Extrinsic stressors were incorporated into the scenarios in the following manner: The patient demonstrated lack of confidence in the student's competency and demonstrated moderately aggressive behavior, such as reluctance to consult, impatience, and manifestations of negative mood. These extrinsic stressful conditions were applied to scenario A or B, according to the group assignment. This condition was called "aggressive patient", and the opposite condition was called "pleasant patient".

As such, the following conditions were created:

1) IS \& ES: scenario A (high clinical severity) with an aggressive patient;

2) IS \& no ES: scenario A (high clinical severity) with a pleasant patient;

3) ES \& no IS: scenario B (low clinical severity) with an aggressive patient;

4) No ES \& no IS: scenario B (low clinical severity) with a pleasant, cooperative patient.

In the first study scenario, students were randomly assigned to one of these four groups. In the second study scenario, students were allocated to the group having the opposite characteristics. Thus students were assigned to either a) condition 1 followed by condition 4 , b) condition 2 followed by condition 3 , c) condition 4 followed by condition 1 , or d) condition 3 followed by condition 2 .

\section{Standardized Patient (SP) Training and Simulation of Ambulatory Setting}

Eight professional actors were hired from a local theatre company. During a two-hour training session for each scenario, the actors were given a detailed description of the patient symptoms and character. The SPs were also trained regarding their demeanor for both the low-stress and the high-stress scenarios. Each item of the clinical skills checklist was carefully explained in order to describe the expected skill. The objective of the explanations was to teach the SPs to distinguish between different levels of performance and to accurately complete the Likert scales according to three levels (done, not done or partially done). For communication skills assessment, levels 0 and 100 of each analogical scale were described with behavioral anchors. All medical terms were described in detail with non-medical language, and any remaining questions were addressed to the satisfaction of the SPs.

The experiences were performed in an authentic environment, which included a desk, chairs, telephone, computer, and examination devices such as stethoscope, blood pressure cuff, and reflex hammer.

\section{Assessment of Clinical Performance}

Five components of clinical performance were assessed. Two of them were assessed by the SPs (see appendix):

1) Clinical abilities score (CLA): At the end of each experience, the SPs completed a checklist regarding the student's performance in obtaining the clinical history and in performing the physical examination. The checklist contained 19 items for scenario A and 24 for scenario B. Each item was scored 0 (not done), 0.5 (partially done) or 1 (entirely done). The items of the checklist were established by two expert clinicians who were specialists in internal medicine, These two scores were transformed into a percentage of maximal total scores. In a previous study ${ }^{10}$ using the same assessment, the correlation coefficient between medical teachers and SPs and between two SPs of the same group of actors were 0.81 and 0.78 , respectively.

2) Communication score (COS): The students' communication skills were evaluated by the SPs on four analogical scales that assessed empathy, discourse coherence, and verbal and nonverbal communication. ${ }^{24}$ A global communication score was generated using the mean of the four items.

For this score, the correlation coefficients between medical teachers and SPs and between two SPs of the same group of actors were 0.58 and 0.6 , respectively, in a previous study. ${ }^{10}$ Three components of clinical performance were assessed using written assessment forms completed by the student immediately following the experience and scored by one of the investigators based on the method described by LeBlanc ${ }^{25}$ :

1) Diagnostic accuracy score (DA): Students were asked to indicate their clinical diagnosis for each experience. Diagnostic accuracy was scored on a three-point Likert scale (false or no diagnosis, partially correct, correct).

2) Positive arguments score (PAS): Students were asked to report the relevant clinical information supporting their main diagnosis. The maximum score was 5 for scenario A and 5 for scenario B.

3) Differential arguments score (DAS): Students were asked to report all relevant clinical information supporting differential diagnoses. The maximum was 4 for scenario $\mathrm{A}$ and 7 for scenario B. 
The items used for scoring were selected by two expert clinicians who were specialists in internal medicine. These scores were transformed into a percentage of maximal total scores. The inter-rater correlations of these three latter scores were $0.92,0.67$ and 0.89 , respectively, in a previous study. ${ }^{10}$

\section{Subjective Stress Measures}

Medical students' subjective stress levels were assessed using three measures:

- A cognitive appraisal (threat/challenge) score (CA $)^{26}$ was assessed before and after each experience by calculating the ratio of primary appraisal (perceived demands) to secondary appraisal (perceived resources) for each student. Primary and secondary appraisal were respectively evaluated by the following questions, translated in French: "How demanding do you expect (or did you feel) the upcoming (or previous) task to be?" and "How are you able to (or did you) cope with this task?" Responses were recorded using a seven-point Likert-type scale. Threat appraisal was defined by a ratio of demands to resources greater than 1 . In laboratory studies, threat appraisals have been associated with greater physiological stress responses than challenge appraisals. ${ }^{27}$

- A visual analog scale (VAS) asking "quantify your stress from 0 (not stressed) to 100 (extremely stressed) on the following visual analog scale" was completed by the students before and after each experience. Such scales have been used as a marker of subjective stress in previous research $^{1,10}$

- The French version of the State-Trait Anxiety Inventory (STAI), developed by Bruchon-Schweitzer ${ }^{28}$ measures anxiety experienced at a given moment. It includes 20 items scored from 1 to 4 on a Likert scale, with a total score ranging from 20 to 80 . High internal consistency of the STAI (Cronbach's $\alpha$ coefficient, 0.92) has been demonstrated ${ }^{29}$

\section{Potential Confounders}

Previous research has shown that sex can influence both responses to stressors and the effects of stress responses on measures of cognitive ability. ${ }^{30}$ As such, we recorded each student's sex, with men coded as 1 and women as 2. As the inclusion of the stressors could potentially affect the difficulty level of the experiences, the student-perceived cognitive difficulty of the scenarios was assessed $5 \mathrm{~min}$ after each experience using a visual analogical scale ranging from 0 ("very easy") to 100 ("very difficult"). The question was the French equivalent of "From the clinical reasoning perspective, how difficult did you find this scenario?"

\section{Sample Size}

Given the absence of previous studies with similar designs and outcomes, no sample size calculation was performed. Thus the study was open to all third-year medical students at the University of Nantes (France).

\section{Statistical Analysis}

The three subjective stress measures (STAI, VAS, CA) and the five clinical performance measures described above were compared using multivariate mixed analyses of variance (ANOVA), with time (T2, T3) as the repeated measure and the following variables as fixed effects: baseline value (if available), presence of extrinsic and/or intrinsic stressors, sex, and student-perceived cognitive difficulty. Time and baseline value effects were imposed in the models even if these effects were not significant. In each model, the interactions between extrinsic and intrinsic stressors were tested. Significant effects were selected using a backward selection method. Results are presented in terms of adjusted mean differences on each studied variables ( $\beta$ parameters). For binary variables (time, sex, ES, IS) and for interactions ES x IS, they represent adjusted mean differences on each studied variable between the two groups of students (males and females, for example). For continuous variables (baseline, cognitive difficulty), they represent the gain or loss on each studied variable when the continuous variable increased by one unit. The signs of the $\beta$ parameter correspond to the notion of increase $(+)$ or decrease $(-)$ of each studied variable between the groups coded 0 (females, no IS, no ES) and the groups coded 1 (males, presence of IS, presence of ES) or when a continuous variable increased by one unit. The significance level was fixed at $5 \%$. Statistical analyses were conducted using Stata 10 software.

\section{RESULTS}

One hundred nine students $(49.5 \%$ men, mean age $21.6+/-$ 0.8 years) from a population of 236 third-year students participated in the study (response rate $46 \%$ ). There were no differences between participants and non-participants in terms of sex $(p=0.23)$, first-year ranking $(p=0.30)$, or number of registrations at university before the third year $(p=0.06)$. Participants were, on average, 4 months younger than nonparticipants $(20.6+/-0.8$ vs. $21.0+/-2.1, p=0.04)$. There were no differences among the four groups of study participants in terms of mean age, sex, year 1 ranking, or pre-study measures of stress.

Means and confidence intervals according to the four stress conditions and time are displayed without any covariate adjustments in Table 1. Table 2 shows the independent effects of ES and IS, taking into account potential confounding variables. 


\section{Effects of Extrinsic Stressor (ES) and Intrinsic Stressor (IS)}

Multivariate analysis showed that the presence of ES led to increased scores on the post-scenario STAI ( $\beta$ [regression parameter $]=3.9, p=0.003$ ) and higher scores on the two clinical skills measures: clinical abilities $(\beta=12.3, p<0.001)$ and communication score $(\beta=15.4$, $p<0.001)$. The presence of ES had no effect on the measures of clinical reasoning.

The presence of IS had no effect on either the stress or clinical skills measures. The presence of IS led to increases in diagnostic accuracy $(\beta=9.70, p=0.004)$ and differential argumentation scores $(\beta=5.92, p=0.02)$. The presence of IS conjointly with ES canceled the positive effect of ES, as reported above, for clinical abilities $(p=0.16)$ and communication score $(p=0.11)$ (data not shown).

\section{Effect of Confounding Variables (Time, Sex, Order, Student-Perceived Cognitive Difficulty)}

Students' VAS and STAI scores, but not their CA scores, decreased over time $(\beta=-7.3, p<0.001$ and $-4.1, p<0.001$, respectively). Time had no effect on any of the performance measures. Communication skills scores were higher in female than in male students $(\beta=-4.9, p=0.04)$. An unexpected high level of student-perceived cognitive difficulty was found for both scenarios, but especially for scenario A (mean score of $46.5+/-21.0$ for scenario B and $63.3+/-20.4$ for scenario A), which had a substantial impact on stress and performance measures, with the exception of clinical abilities and communication scores (cf. Table 2). No effect was found for the order in which the students were exposed to intrinsic and extrinsic stressors.

\section{DISCUSSION}

The objective of this study was to evaluate the effects of intrinsic and extrinsic stressors on subjective stress responses and clinical performance of medical students. The results reveal that the student-perceived cognitive difficulty of the task emerged as a strong predictive factor for most of the stress and performance variables. When the effects of cognitive difficulty were accounted for in the analyses, the presence of extrinsic stressors increased subjective stress and improved clinical skills (clinical abilities and communication), while the presence of intrinsic stressors increased diagnostic accuracy and differential argumentation (i.e., the ability to evoke alternative diagnoses), without affecting stress responses. Furthermore, the presence of intrinsic stressors negated the beneficial effects of extrinsic stressors on clinical abilities when both were present. When presented together, ES and IS led to distress, while ES and IS alone 
Table 2 Stress and performances measures-results of multivariate analysis $(n=109)$

\begin{tabular}{|c|c|c|c|c|c|c|c|c|c|c|}
\hline & & & Time & Baseline & Sex & $\begin{array}{l}\text { ES } \\
\text { aggressiveness }\end{array}$ & $\begin{array}{l}\text { IS clinical } \\
\text { severity }\end{array}$ & $\begin{array}{l}\text { Cognitive } \\
\text { difficulty }\end{array}$ & $\begin{array}{l}\text { Interaction } \\
\text { IS*ES }\end{array}$ & intercept \\
\hline \multirow[t]{5}{*}{ Stress measures } & VAS & $\begin{array}{l}\beta \\
p^{*}\end{array}$ & $\begin{array}{l}-7.26 \\
<0.001\end{array}$ & $\begin{array}{c}0.45 \\
<0.001\end{array}$ & ${ }^{-} 0.41$ & 0.73 & - & $\begin{array}{l}0.15 \\
0.006\end{array}$ & \multirow{2}{*}{0.89} & $\begin{array}{c}14.22 \\
0.017\end{array}$ \\
\hline & \multirow[t]{2}{*}{ STAI } & $\beta$ & -4.12 & 0.52 & - & 3.88 & - & 0.15 & & 10.82 \\
\hline & & $\mathrm{p}^{*}$ & $<0.001$ & $<0.001$ & 0.13 & 0.003 & \multirow{2}{*}{0.43} & $<0.001$ & \multirow{2}{*}{0.23} & 0.002 \\
\hline & \multirow[t]{2}{*}{$\mathrm{CA}$} & $\beta$ & 0.02 & 0.54 & - & \multirow[t]{2}{*}{ - } & & \multirow{2}{*}{$<0.001^{0.02}$} & & -0.62 \\
\hline & & $\mathrm{p}^{*}$ & 0.85 & $<0.001$ & 0.62 & & 0.69 & & 0.13 & 0.001 \\
\hline \multirow{4}{*}{ Clinical skills measures } & \multirow{2}{*}{ CLA } & $\beta$ & -1.07 & - & - & 11.86 & -15.38 & \multirow{2}{*}{$\begin{array}{l}- \\
<0.001\end{array}$} & $\begin{array}{r}0.13 \\
14.52\end{array}$ & \multirow{2}{*}{40.67} \\
\hline & & $\mathrm{p}^{*}$ & 0.47 & 0.76 & $<0.001$ & $<0.001$ & 0.17 & & $<0.001$ & \\
\hline & \multirow{2}{*}{ COS } & $\beta$ & 0.66 & - & -4.91 & 16.24 & -11.03 & & 12.79 & \multirow[t]{2}{*}{64.78} \\
\hline & & $\mathrm{p}^{*}$ & 0.72 & 0.037 & $<0.001$ & $<0.001$ & 0.074 & $<0.001$ & $<0.001$ & \\
\hline \multirow{6}{*}{$\begin{array}{l}\text { Clinical reasoning } \\
\text { measures }\end{array}$} & \multirow[t]{2}{*}{ DA } & $\beta$ & 2.19 & - & - & - & 9.70 & -0.31 & & \multirow[t]{2}{*}{56.51} \\
\hline & & $\mathrm{p}^{*}$ & 0.48 & 0.98 & 0.39 & 0.004 & $<0.001$ & 0.83 & $<0.001$ & \\
\hline & \multirow[t]{2}{*}{ PAS } & $\beta$ & -5.37 & - & - & - & - & -0.45 & & \multirow{2}{*}{72.62} \\
\hline & & $\mathrm{p}^{*}$ & 0.15 & 0.27 & 0.48 & 0.056 & $<0.001$ & 0.32 & $<0.001$ & \\
\hline & \multirow[t]{2}{*}{ DAS } & $\beta$ & -3.02 & - & & - & 5.92 & -0.14 & & \multirow[t]{2}{*}{25.10} \\
\hline & & $\mathrm{p}^{*}$ & 0.18 & 0.48 & 0.62 & 0.016 & 0.001 & 0.58 & $<0.001$ & \\
\hline
\end{tabular}

VAS visual analogical scale, STAI Spielberger State-Trait Anxiety Inventory, CA cognitive appraisal, CLA clinical abilities, COS communication skills, $D A$ diagnostic accuracy, PAS positive argumentation score, DAS differential argumentation score, IS intrinsic stressors, ES extrinsic stressors

$\beta:$ regression parameter $=$ adjusted mean differences :

-between the two groups for binary variables (sex, ES, IS)

- for an increase of 1 unity for continuous variables (baseline, cognitive difficulty)

* multivariate mixed analysis of variance

led to eustress. It is also important to note that since we found no effect of the order of exposure to IS and ES, the effect caused by the presence of a stressor was of short duration. These results suggest that intrinsic and extrinsic stressors have different effects on subjective stress responses and on clinical skills and clinical reasoning abilities of medical students.

Some hypotheses can be put forward to explain the main findings (cf. Fig. 2) emerging from our multivariate analysis. The different effects of the intrinsic and extrinsic stressors can

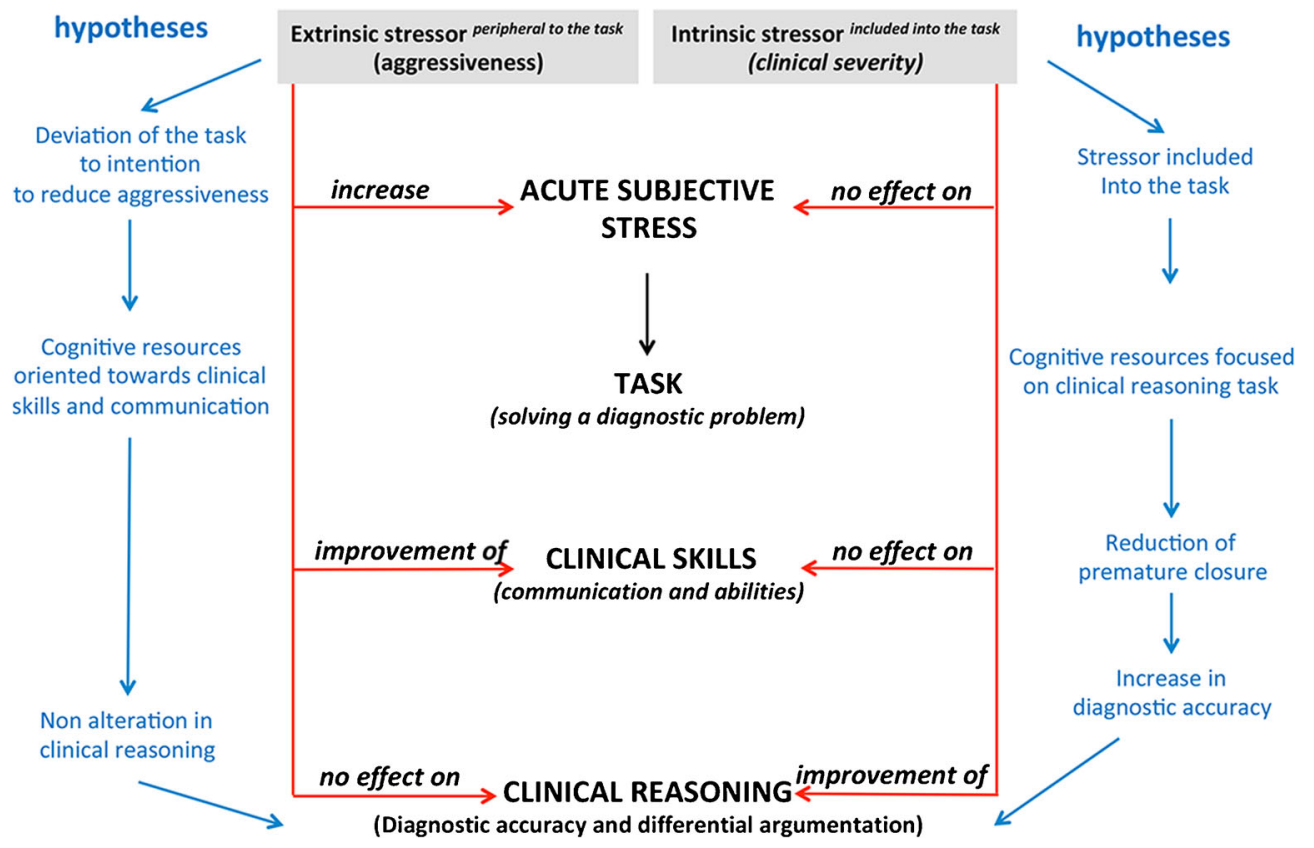

Fig. 2 Main findings and hypotheses 
be explained based on the construct that stressors capture, or bias, cognitive resources. ${ }^{12}$ Intrinsic stressors, being intrinsically linked to a task, serve to focus cognitive resources towards the task (solving a clinical problem), which may explain the increase in diagnostic accuracy and differential argumentation. In contrast, the extrinsic stressors, the patient's aggressive demeanor, likely oriented students' cognitive resources towards the interpersonal aspects of the task, which in turn may result in increased communication and clinical abilities. It was as if a trainee, confronted with an aggressive patient, tried to demonstrate and make explicit his/her clinical abilities, which are competencies widely agreed as inherent to a good health professional.

The theory of cognitive load or working memory limitations $^{31}$ could explain that in our novice medical students, under IS, clinical reasoning was enhanced at the expense of clinical skills, and conversely, under ES, clinical skills were enhanced at the expense of clinical reasoning.

Our study is not the first to report an advantageous effect of stressors on clinical performance. In a reanimation setting, DeMaria $^{15}$ found long-term improvement of clinical performance in students who had been exposed to extrinsic stressors 6 months before. LeBlanc also found improved performance under extrinsic stress conditions with emergency medicine ${ }^{22}$ and surgery residents. ${ }^{8}$ One possible explanation for these results is that under moderately stressful conditions, some cognitive functions such as memory and reasoning may be selectively enhanced, leading to an increase in some aspects of performance. Indeed, Buchanan ${ }^{6}$ found that the inclusion of stressors that did not induce a cortisol response led to enhanced memory retrieval, while cortisol responses were accompanied by impaired performance. Domes ${ }^{7}$ also showed an inverted-U type of performance curve as a function of cortisol response. In that study, mild responders showed higher performance than non- or high responders.

The results of the study should be interpreted within the context of several limitations. First, the effect of perceived cognitive difficulty was greater than expected. Although some of these effects could be accounted for statistically, it nevertheless could have disturbed the manipulations of intrinsic and extrinsic stressors and masked part of their effects. Second, although we selected common and previously taught clinical conditions for this study, the relatively high perceived cognitive difficulty suggests that the students may have lacked some knowledge for dealing with the scenarios, which may have influenced the observed effects of the studied stressors. Third, the generalizability of our findings is limited by the small number of scenarios and the fact that we chose to focus on a single variable potentially affecting clinical performance (i.e., acute stress). In real settings, clinical performance involves far more complex, interactive processes likely to alter the effects found in this study. Fourth, though the conceptual difference between ES and IS was clear, in our study the boundary between them was probably more tenuous. A questionnaire designed to ascertain whether students actually appraised IS and ES as expected might have been informative. Fifth, the student-perceived cognitive difficulty of the task was assessed with a single global rating immediately after the encounter, concurrently with stress measurement, which may have been insufficient to correctly assess this dimension and could have induced confusion with students' overall stress.

In conclusion, this study showed that in the context of standardized patients, both extrinsic and intrinsic stressors had positive effects on clinical performance, but on different aspects. The results are consistent with the concept that stressors serve to capture cognitive resources and orient them towards sources of potential threat. Finally, our findings suggest that under stress conditions, medical students resort to an increased panel of clinical skills. Given that recent studies have demonstrated that stressful events are likely to enhance long-term memory and learning, ,3,15,32 an implication for clinical training is that regularly exposing students to ES and IS may be a useful educational tool for the long-term development of their clinical abilities and reasoning. Future research might look at the longer-term effects of exposing medical students to ES and IS. As was recommended in a recent review of the literature, ${ }^{33}$ such a study would inform the body of knowledge for a broader area of research investigating the effect of emotional state on cognitive processes.

\begin{abstract}
Acknowledgments: The authors wish to acknowledge Professor Jean-Michel Rogez, Dean of the Nantes University Medical School, for his moral and logistic support. We also wish to thank the following researchers in health professional education for their valuable scientific revisions: Dominique Piquette, Carilynne Yarascavitch, and Walter Tavares. Financial support for this study was provided by the the Urgo Corporate Foundation, Janssen Pharmaceutical laboratory, the Roche Foundation, Equip' Santé Biron, Midmark Corporation, MACSF (Mutuelle d'Assurance du Corps de Santé Français), Medicalem, and ADREMSE(Association pour le développement de la recherche en médecine et en sciences de l'éducation). This work was presented as an ePoster, "Variations of subjective and physiological stress and clinical reasoning according to extrinsic and intrinsic stressors", at the Association for Medical Education in Europe (AMEE) conference in Prague, September 2013.
\end{abstract}

Conflict of Interest: The authors declare that they do not have a conflict of interest.

Corresponding Author: Pierre Pottier, MD PhD; Department of Internal Medicine, University Hospital Center, Faculty of Medicine, University of Nantes, CHU Nantes, Place Alexis Ricordeau, Nantes 44093, France (e-mail: pierre.pottier@univ-nantes.fr).

\section{REFERENCES}

1. Pottier P, Hardouin JB, Desjoie T, et al. Stress responses in medical students in ambulatory and in-hospital patient consultations. Med Educ. 2011;45:678-87.

2. Lazarus RS, Folkman S. Stress Appraisal and Coping. 1st ed. New York: Springer; 1984 
3. LeBlanc VR. The effects of acute stress on performance: implications for health professions education. Acad Med. 2009;10:S25-S33.

4. Chajut R, Algom D. Selective attention improves under stress: Implications for theories of social cognition. J Pers Soc Psychol. 2003; $85: 231-48$

5. Elzinga BM, Roelofs $\mathbf{K}$. Cortisol-induced impairments of working memory require acute sympathetic activation. Behav Neurosci. 2005;119:98-103.

6. Buchanan TW, Tranel D. Stress and emotional memory retrieval: Effects of sex and cortisol response. Neurobiol Learn Mem. 2008;89:134-41.

7. Domes G, Heinrichs M, Rimmele U, Reichwald U, Hautzinger M. Acute stress impairs recognition for positive words association with stress induced cortisol secretion. Stress. 2004;7:173-81.

8. LeBlanc VR, Woodrow SI, Sidhu R, Dubrowski A. Examination stress leads to improvements on fundamental technical skills for surgery. Am J Surg. 2008;196:114-9.

9. Johnston JH, Driskell JE, Salas E. Vigilant and hypervigilant decision making. J Appl Psychol. 1997;82:614-22.

10. Pottier P, Dejoie T, Hardouin JB, et al. Effect of stressful scenarios in simulated consultation on stress and diagnostic judgment. Med Teach. 2013;35:472-80.

11. Graber ML, Franklin N, Gordon R. Diagnostic error in internal medicine. Arch Int Med. 2005;165:1493-9.

12. MacLeod C, Rutherford EM. Anxiety and the selective processing of emotional information: Mediating roles of awareness, trait and state variables, and personal relevance of stimulus materials. Behav Res Ther. 1992;30:479-91.

13. Harvey A, Nathens AB, Bandiera G, LeBlanc VR. Threat and challenge: cognitive appraisal and stress responses in simulated trauma resuscitations. Med Educ. 2010;44:587-94.

14. Patil PG, Apfelbaum JL, Zacny JP. Effects of a cold-water stressor on psychomotor and cognitive functioning in humans. Physiol Behav. 1995;58: 1281-6.

15. DeMaria S, Bryson EO, Mooney TJ, Silverstein JH, Reich DL, Bodian C, Levine AL. Adding emotional stressors to training in simulated cardiopulmonary arrest enhances participant performance. Med Educ. 2010;44:1006-15.

16. Liu CC, Wissow LS. Residents who stay late at hospital and how they perform the following day. Med Educ. 2008;42:74-81.

17. Kirschbaum C, Pirke KM, Hellhammer DH. The "Trier Social Stress Test" - a tool for investigating psychobiological stress responses in a laboratory setting. Neuropsychobiology. 1993;28:76-81.

18. Van Dulmen S, Tromp F, Grosfeld F, ten Cate O, Bensing J. The impact of assessing simulated bad news consultations on medical students' stress response and communication performance. Psychoneuroendocrinology. 2007;32:943-50.
19. Brown R, Dunn S, Byrnes K, Morris R, Heinrich P, Shaw J. Doctor's stress responses and poor communication performance in simulated badnews consultations. Acad Med. 2009;84:1595-602.

20. LeBlanc VR, Mac Donald RD, Mc Arthur B, King $K$, Lepine $T$. Paramedic performance in calculating drug dosages following stressful scenarios in a human patient simulator. Prehosp Emerg Care. 2005;9:439-44.

21. Gimmig D, Huguet P, Caverni JP, Cury F. Choking under pressure and working memory capacity: when performance pressure reduces fluid intelligence. Psychon Bull Rev. 2006;13:1005-10.

22. LeBlanc VR, Bandiera GW. The effect of examination stress on the performance of emergency medicine residents. Med Educ. 2007;41:55664.

23. Bovier PA, Perneger TV. Stress from uncertainty from graduation to retirement. A population-based study on Swiss physicians. J Gen Int Med. 2007;22:632-8.

24. Hodges B, Mc Ilroy JH. Analytic global OSCE ratings are sensitive to level of training. Med Educ. 2003;37:1012-6.

25. LeBlanc VR, Brooks LR, Norman GR. Believing is seeing: the influence of a diagnostic hypothesis on the interpretation of clinical features. Acad Med. 2002;77:S67-9.

26. Tomaka J, Blaskovich J, Kelser RM, Leitten CL. Subjective, physiological and behavioral effects of threat and challenge appraisal. J Pers Soc Psychol. 1993;65:248-60.

27. Dickerson S, Kemeny ME. Acute stressors and cortisol responses: a theoretical integration and synthesis of laboratory research. Psychol Bull. 2004; 130:355-91.

28. Bruchon-Schweitzer M, Paulhan I. Manuel français de l'échelle d'anxiété-trait. Anxiété état de CD, Spielberger, Paris, France, ECPA; 1993.

29. Spielberger CD, Gorsuch RL, Lushene R, Vagg PR, Jacobs GA. Manual for the State-Trait Anxiety Inventory. Palo Alto, CA: Consulting Psychologists Press; 1983.

30. Stroud LR, Salovey P, Epel ES. Sex differences in stress responses: social rejection versus achievement stress. Biol Psychiatry. 2002;52:318-27.

31. Young JQ, Van Merrienboer J, Duming S, ten Cate O. Cognitive load theory. Implications for medical education. AMEE Guide $\mathrm{N}^{\circ}$. 86. Med Teach. 2014;36:371-84.

32. Kromann CB, Jensen ML, Ringsted C. Test-enhanced learning may be a gender-related phenomenon explained by changes in cortisol level. Med Educ. 2011;45:192-9.

33. LeBlanc VR, McConnell MM, Monteiro SD. Predictable Chaos: A Review of the Effects of Emotions on Attention, Memory and Decision Making. Adv Health Sci Educ. 2014. doi:10.1007/ s10459-014-9516-6. 


\section{APPENDIX : CLINICAL ABILITIES CHECKLIST USED BY STANDARDIZED PATIENTS}

SCENARIO A (pulmonary embolism)

The student asked for :

onset of dyspnea (acute or not)

bed rest or loss of walking

loss of weight

preexisting breathlessness during effort

malignancy history

thoracic pain and cough and sputum

pain in the leg

breathlessness lying at night

fever

\section{HISTORY TAKING}
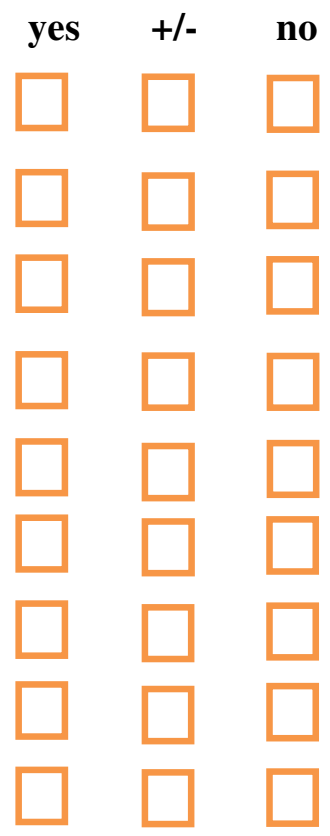

\section{PHYSICAL EXAMINATION}

\section{The student :}

assessed respiratory rythm (hand on thorax)

looked carefully at the fingers (cyanosis)

looked for asterixis (hands up during 30s)

looked for a liver-jugular reflux

looked carefully at the legs

palpated the legs

assessed temperature

assessed cardiac frequency and blood pressure

auscultated the lungs and the heart

looked carefully at the conjunctival membrane
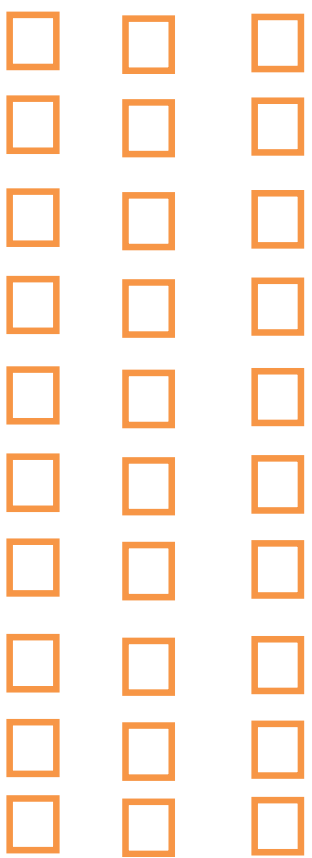


\section{SCENARIO B (an acute pancreatitis)}

\section{HISTORY TAKING}

The student asked for :

oui +/- non

Onset of pain (acute or not)

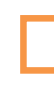

localisation of pain

irradiations of pain

triggering factors (meal, alcool)

easing factors (meal, treatments, position)

diarrhea, nausea, vomiting

nature of the pain (burning sensation, torsion..)

loss of weight

colouring of feces and urine

chronic diarrhea

chronic extensive pruritus

history of cholecystectomy

history of abdominal pains
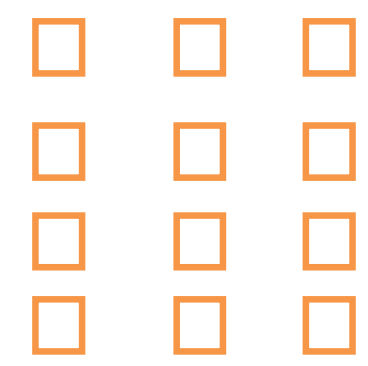

$\square$
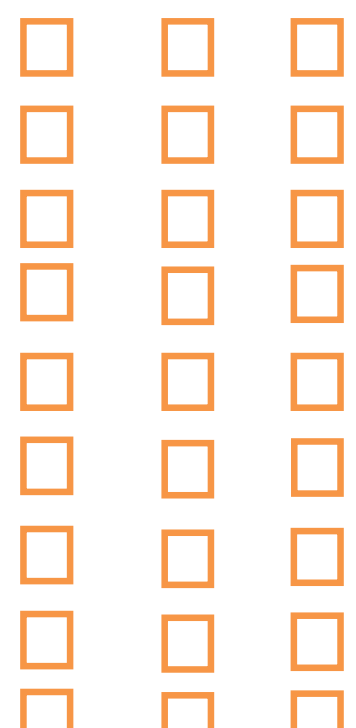

usual intake of alcool

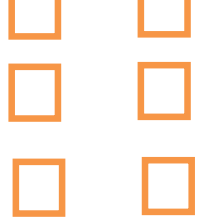




\section{The student :}

\section{PHYSICAL EXAMINATION}

looked carefully at the abdomen (scars)

palpated hernial orifices

palpated the abdomen

looked for an epigastric pain at palpation

palpated and looked under the last rib

looked carefully at conjonctival membranes

palpated and looked at the knees

assessed temperature

assessed cardiac frequency and blood pressure

percussed the abdomen

looked for a lymph node above the clavicle

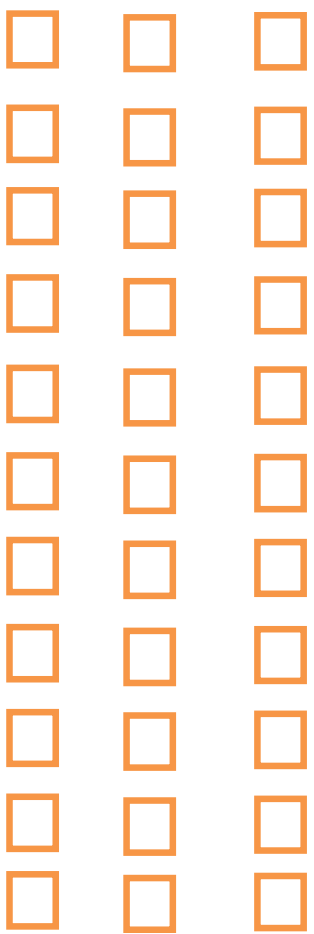

